\title{
A Case of Brucellosis Following Kidney Transplantation
}

Eun Hwa Choi ${ }^{1,2}$, Hee-Sup Kim ${ }^{3}$, Hoan Jong Lee ${ }^{1,2}$

${ }^{1}$ Department of Pediatrics, Seoul National University Children's Hospital

${ }^{2}$ Department of Pediatrics, Seoul National University College of Medicine, Seoul, Korea

${ }^{3}$ Department of Pediatrics, Dongguk University Ilsan Hospital, Goyang, Korea

\section{Background}

- Brucellosis is one of the most common systemic zoonotic diseases transmitted by consumption of unpasteurized dairy products or by occupational contact with infected animals. Brucellosis is very rare in renal transplant recipients.

\section{Case summary}

- A 9-year-old Emirati girl was brought to the emergency room of Seoul National University Children's Hospital (SNUCH) because of 1 day fever at Nov $7^{\text {th }}, 2017$. On July 26th, 2017, the patient who were diagnosed with chronic kidney disease from her congenital nephrotic syndrome at UAE came to Korea to undergo kidney transplantation at $\mathrm{SNUCH}$.

She received the kidney transplant from her mother at August 23th, 2017

- In the UAE, her family owns a camel and sheep farms that are located far away from their house. The patient has never been to the farms and denied any direct contact with the animals. Her family often ate cooked sheep meat and occasionally boiled and drank raw camel milk usually during the Ramadan.

The patient did not remember the first time she drank the camel milk but she drank about nine times, and the most recent time the family had the milk was four months prior the admission (June 2017).

- Two months prior to visit, on the first week of September, some of the family members including the patient's mother started to have mild fever, abdominal pain and anorexia.
The mother's fever persisted for 3 weeks, so she was admitted (September 25th) and initially treated with intravenous ceftriaxone and metronidazole under the impression of peritonitis.

On the 8th day of her mother's admission, Brucella melitensis grew on her initial blood cultures.

Antibiotics were changed to doxycycline, rifampin, and ciprofloxacin, and her blood culture became negative on the 9th day of

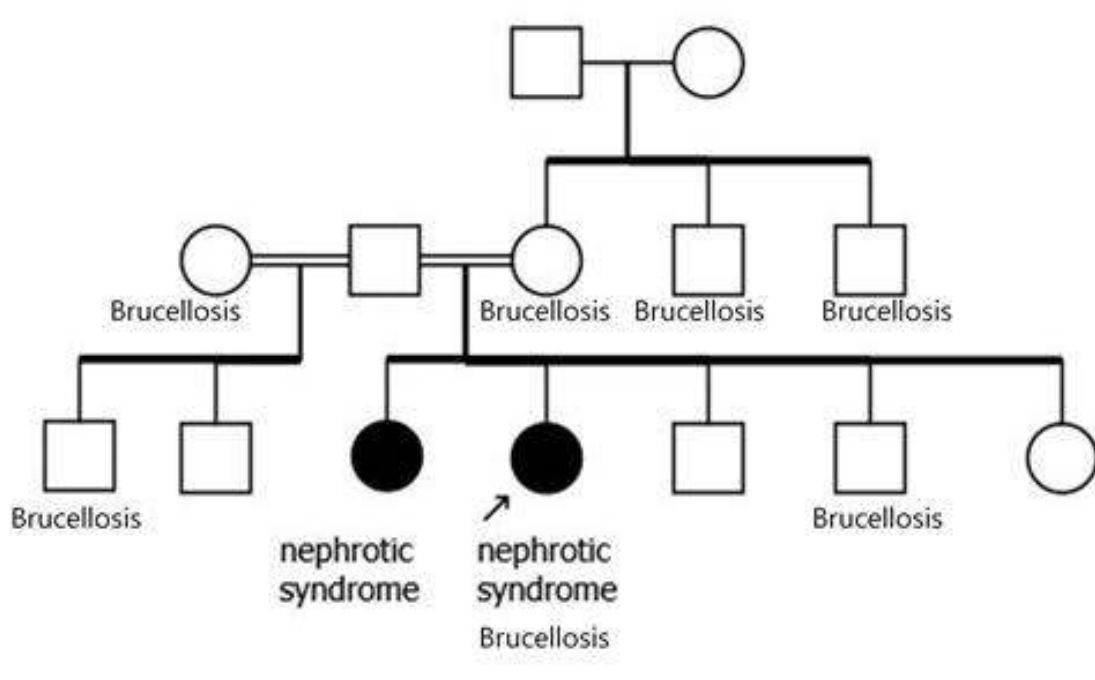

- In the ER at initial presentation (22th October), our patient had fever that reached up to $39.0^{\circ} \mathrm{C}$. She did not have any other symptoms.

- She was not ill-looking and physical findings including those on abdomen and surgical site were all normal.

\section{Clinical course}

- HD2 (10/24): Therapy for Brucellosis was started with oral doxycycline and rifampin. HD3 (10/25): Gram (-) rod was growing on all two sets of her initial blood cultures.

- HD4 (10/26): Gentamycin was added.

- HD7 (10/28): Creatinine level rose up to $4.6 \mathrm{ng} / \mathrm{ml}$. Rifampin and gentamycin were discontinued due to the interaction with tacrolimus and nephrotoxicity, respectively. Oral trimethoprim-sulfamethoxazole was added.

\begin{tabular}{|c|r|r|r|r|r|r|}
\hline Test & $\begin{array}{c}\text { ER } \\
(\mathbf{1 0 / 2 2})\end{array}$ & $\begin{array}{c}\text { HD4 } \\
(\mathbf{1 0 / 2 6})\end{array}$ & $\begin{array}{c}\text { HD6 } \\
\mathbf{( 1 0 / 2 8 )}\end{array}$ & $\begin{array}{c}\text { HD8 } \\
(\mathbf{1 0 / 3 0})\end{array}$ & $\begin{array}{c}\text { HD10 } \\
\mathbf{( 1 1 / 0 1})\end{array}$ & $\begin{array}{c}\text { HD12 } \\
(\mathbf{1 1 / 0 3})\end{array}$ \\
\hline Serum WBC $\left(/ \mathrm{mm}^{3}\right)$ & 9,690 & 5,000 & 9,030 & 6,490 & 10,670 & 14,350 \\
\hline CRP $(\mathrm{mg} / \mathrm{dl})$ & 2.4 & 3.9 & 10.3 & 2.9 & 1.7 & 0.6 \\
\hline BUN (mg/dl) & 37 & 26 & 57 & 82 & 50 & 55 \\
\hline Creatinine (mg/dl) & 1.18 & 2.03 & 4.69 & 3.49 & 1.81 & 1.69 \\
\hline Urine WBC (/HPF) & $5-9$ & $5-0$ & $20-29$ & $5-9$ & $10-19$ & $10-19$ \\
\hline Albumin & \pm & $3+$ & $3+$ & $1+$ & $1+$ & $1+$ \\
\hline Glucose & - & - & - & - & \pm & $1+$ \\
\hline
\end{tabular}

- HD8 (10/29): The growth of $B$. melitensis on initial blood cultures was identified.

- HD9 (10/30): Tacrolimus dose was increased to $5 \mathrm{mg}$ bid.

- HD21 (11/12): Doxycycline dose was increased from $50 \mathrm{mg}$ bid to $100 \mathrm{mg}$ bid.

- HD24 (11/15): Fifth blood culture was identified as 7 days no growth, and the patient discharged from hospital.

- Outpatient clinic (12/18): Antimicrobial therapy was completed.

\section{Discussion}

- The patient was managed with oral doxycycline and rifampin/TMP-SMX for 8 weeks and completely recovered without complications.

- Although brucellosis very rare after kidney transplant, for both recipients and donors from endemic areas, it should be considered as it can mimic other infectious diseases.

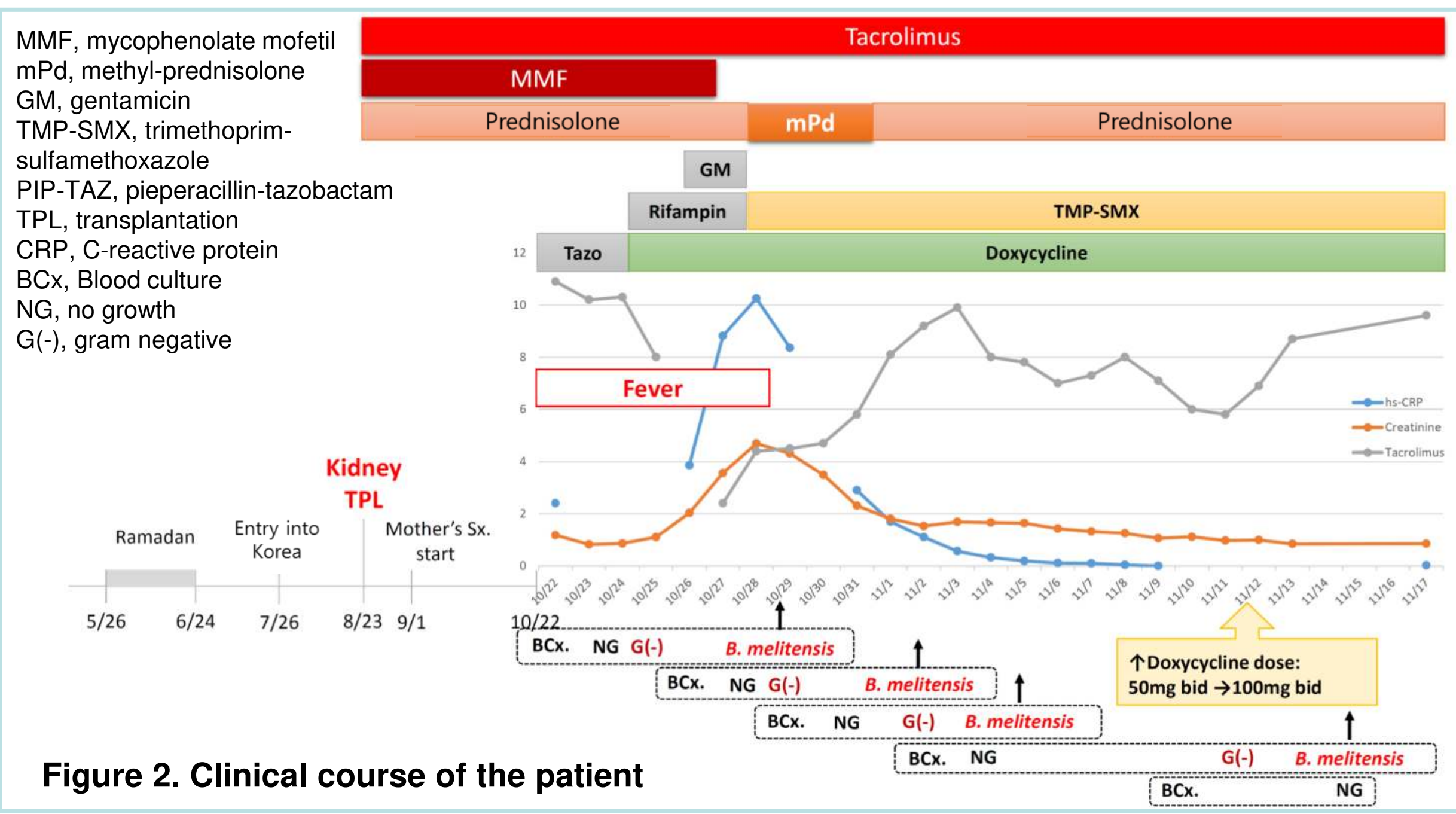

